\title{
Analytical solutions of incompressible laminar channel and pipe flows driven by in-plane wall oscillations ${ }^{\circledR}$
}

Cite as: Phys. Fluids 31, 083605 (2019); https://doi.org/10.1063/1.5104356

Submitted: 26 April 2019 . Accepted: 17 July 2019 . Published Online: 12 August 2019

Tamás István Józsa (iD)

COLLECTIONS

EP This paper was selected as an Editor's Pick

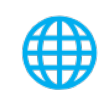

\section{ARTICLES YOU MAY BE INTERESTED IN}

Large-eddy simulation of turbulent natural-bed flow

Physics of Fluids 31, 085105 (2019); https://doi.org/10.1063/1.5116522

Oscillating grid turbulence in shear-thinning polymer solutions

Physics of Fluids 31, 083102 (2019); https://doi.org/10.1063/1.5113551

Single diffusive magnetohydrodynamic pressure driven miscible displacement flows in a channel

Physics of Fluids 31, 082102 (2019); https://doi.org/10.1063/1.5112373

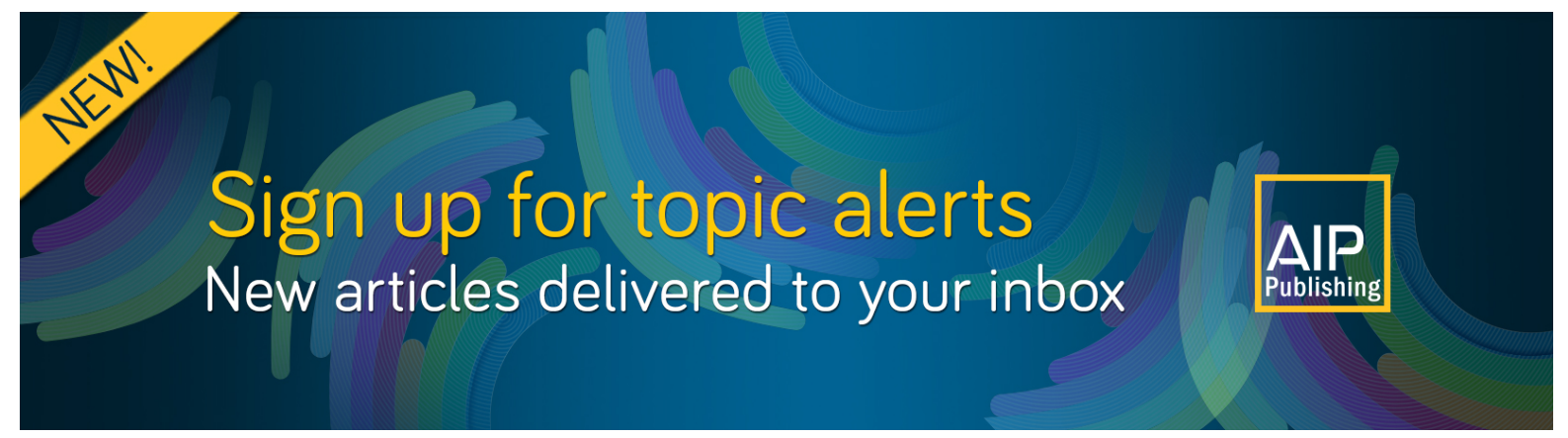




\title{
Analytical solutions of incompressible laminar channel and pipe flows driven by in-plane wall oscillations (ㄹ
}

\author{
Cite as: Phys. Fluids 31, 083605 (2019); doi: 10.1063/1.5104356 \\ Submitted: 26 April 2019 • Accepted: 17 July 2019• \\ Published Online: 12 August 2019
}

Tamás István Józsa ${ }^{\text {a) }}$

\section{AFFILIATIONS}

Institute of Biomedical Engineering, Department of Engineering Science, University of Oxford, Oxford OX1 3PJ, United Kingdom

a) tamas.jozsa@eng.ox.ac.uk

\begin{abstract}
Emerging flow control strategies have been proposed to tackle long-lasting problems, for instance, precise mixing of chemicals and turbulent drag reduction. Employing actuators imposing in-plane wall oscillations are particularly popular. This paper investigates incompressible laminar rectangular channel and circular pipe flows driven by uniform and traveling wave in-plane wall oscillations. A comprehensive set of exact analytical solutions are presented describing parallel and concentric flows. Dimensionless groups are identified, and it is described how they characterize the one- and two-dimensional time-dependent velocity and pressure fields. The solutions enable to compute the oscillating boundary layer thickness. It is demonstrated that the dimensionless groups and the boundary layer thickness narrows the region of interest within the parameter space. In particular, the oscillating boundary layer thickness obtained from these laminar flows estimates a "radius of action" within which flow features can be altered to boost mixing or reduce turbulent friction drag. The results are suitable for software validation and verification, may open the way to promising complex wall oscillations, and ease the optimization task that delays the industrial application of flow controls.
\end{abstract}

Published under license by AIP Publishing. https://doi.org/10.1063/1.5104356

\section{INTRODUCTION}

Due to the rapid development of actuators in recent decades, active flow control has gained much interest in fluid dynamics research. Practical applications include biosensors, ${ }^{1}$ microfluidic mixing, ${ }^{2}$ and turbulent drag reduction. ${ }^{3}$ Controls used in microfluidic systems and turbulent drag reduction often comprise in-plane uniform and traveling wave (tw) wall oscillations, which are the focal points of the present study. Subsection I A provides a literature review of the application of in-plane wall motions for drag reduction purposes. For a concise review of in-plane wall motions used in microfluidic systems and biosensors, the reader is referred to the work of Ricco and Hicks. ${ }^{4}$ Available analytical solutions are reviewed in Subsection I B.

\section{A. Turbulent drag reduction}

Moving fluids through channels and vehicles through fluids dissipates $25 \%$ of the energy used in industrial and commercial environments. ${ }^{5,6}$ Manipulating turbulent boundary layers aims at lowering the friction drag contributing to as much as $\approx 50 \%, \approx 90 \%$, and $\approx 100 \%$ of the aforementioned losses in airliners, marine vehicles, and pipe networks, respectively.

It has been reported, both in numerical and experimental studies, that spatially uniform spanwise wall oscillations ${ }^{8-14}$ and spanwise wall oscillations exhibiting streamwise-traveling waves ${ }^{15-18}$ can lead to turbulent friction drag reduction ranging from 15\% to more than $40 \%$. Low Reynolds number direct numerical simulations predicted $7.3 \%$ peak net energy saving for uniform wall oscillations. ${ }^{11}$ By comparison, $18 \%$ peak net energy saving was reported in the case of spanwise wall oscillations exhibiting streamwise-traveling waves. ${ }^{15}$ These strategies can result in drag reduction and net energy saving even though certain parameters lead to drag increase. ${ }^{19}$ However, active flow controls exploit primarily spanwise wall oscillations because of their higher efficiency compared to streamwise oscillations; ${ }^{20}$ it has been demonstrated that streamwise oscillations of a conceptual compliant coating can reduce turbulent friction drag. $^{21,22}$ 
These flow controls, ${ }^{8-18}$ along with other strategies based on inplane wall motions, ${ }^{20,21,23}$ suppress turbulence production by weakening the quasistreamwise vortices of the near-wall cycle. ${ }^{5,24}$ This drag reduction mechanism is efficient, especially in low Reynolds number turbulent boundary layers. However, it has been recognized that, in the high Reynolds number turbulent regime which is more relevant for industrial applications, large scale and very large scale motions (superstructures) tend to dominate the boundary layer. ${ }^{25-27}$ The contribution of these structures to turbulent friction drag becomes higher with the increasing Reynolds number, 28,29 whereas the near-wall cycle is "squeezed" to a thinning fluid layer. For this reason, the efficiency of flow controls targeting the nearwall cycle drops as the Reynolds number increases. ${ }^{12,21,30}$ The beneficial alteration of the aforementioned superstructures is a major challenge. $^{12,22,31}$

Complex wall motions encapsulating multiple length and time scales would have greater potential to interact favorably with the broad range of turbulent motions compared to monoharmonic oscillations. Wall oscillations incorporating multiple modes have not been considered before due to the high dimension of the related parameter space. The oscillating boundary layer thickness provides information on how far from the wall in-plane wall motions influence turbulent structures. A priori estimation of the oscillating boundary layer thickness could help narrow the parameter space of active controls, ease the resource-intensive optimization task, and further the development of flow controls incorporating wall motions with multiple modes.

\section{B. Analytical solutions}

In the case of uniform spanwise wall oscillations, it was recognized $^{12,32}$ that phase-averaged spanwise velocity profiles in turbulent boundary layers are well approximated with solutions of the laminar Stokes boundary layer. ${ }^{33}$ Whilst solutions of the Stokes boundary layer are known, ${ }^{33}$ analytical solutions of other wall-motiondriven confined flows, such as pipe and channel flows driven by uniform and/or traveling wave wall motions, have not drawn much attention. Only recently analytical solutions have been obtained for streamwise-traveling spanwise wall oscillations superimposed upon the Poiseullie flow in a channel. Ricco and Hicks ${ }^{4}$ solved the resulting nonlinear partial differential equations (PDEs) using asymptotic analysis. In the following, flow problems are referred to as linear if the convective term is zero so that the governing differential equations are linear.

Nonlinear analytical solutions of the governing equations of incompressible Newtonian fluid flow are rare. ${ }^{34}$ Despite this, under certain technical conditions, it is possible to derive novel linear analytical solutions as it has been recently demonstrated. ${ }^{35-37}$ Here, special cases of incompressible confined flows are considered for which exact linear analytical solutions can be derived. The present work aims at providing a comprehensive description of laminar channel and pipe flows driven by uniform and traveling wave wall motions. For completeness, both streamwise and spanwise or azimuthal wall oscillations are investigated. The examined wall motions sustain time and space-periodic parallel, concentric, and helical flows. ${ }^{38,39}$ Previously, only the decay of these flows was examined. ${ }^{40,41}$ The solutions allow to estimate a priori the oscillating boundary layer thickness, a useful variable for turbulence control which helps to identify wall motions that energize a sufficiently thick fluid layer. ${ }^{19}$ In addition, some of the exact analytical solutions can be combined with Poiseullie flows ${ }^{33}$ therefore opening the way to linear stability analysis. $^{42-44}$

Section II discusses the conditions required to obtain linear flow solutions. Sections III and IV present the resulting simplified equations and their solutions for double-periodic channel and periodic pipe flows. It is examined when the solutions can be superimposed (linearly) to other known analytical solutions, such as the pressure-driven Poiseuille, Couette, ${ }^{33}$ and Womersley ${ }^{45}$ flows. The flow fields described by the analytical solutions are investigated in Sec. V focusing on a similarity condition for the oscillating boundary layer thickness.

\section{GOVERNING EQUATIONS}

The governing equations of incompressible Newtonian fluid flow are the continuity and Navier-Stokes momentum equations which may be written in nondimensional vector form as

$$
\nabla \cdot \vec{u}=0
$$

and

$$
\frac{\partial \vec{u}}{\partial t}+\vec{u} \cdot \nabla(\vec{u})=-\nabla p+\frac{1}{\operatorname{Re}} \nabla^{2} \vec{u} .
$$

Here, $\vec{u}$ is the velocity vector, $t$ is the time, $p$ is the pressure, and $\mathrm{Re}$ is the Reynolds number. The Reynolds number is defined as $\operatorname{Re}=u_{c} l_{c} / v$ with $u_{c}$ being a characteristic velocity, $l_{c}$ being a characteristic length, and $v$ being the kinematic viscosity of the fluid.

Figure 1 displays the flow domains of interest. In Fig. 1(a), a rectangular channel with the Cartesian coordinate system is sketched such that wall-normal, spanwise, and streamwise
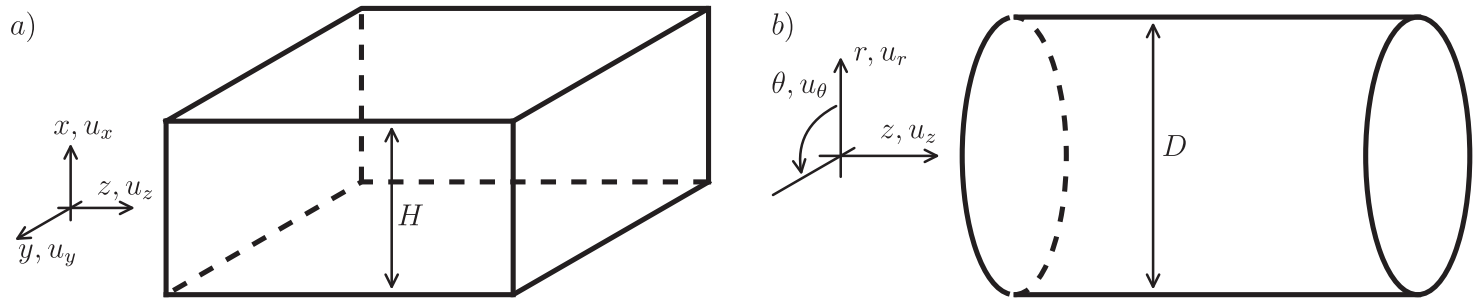

FIG. 1. Drawings of (a) channel flow in a Cartesian coordinate system and (b) pipe flow in a cylindrical coordinate system. The origins are fixed at the center of the geometries. 
coordinates are oriented in $x$-, $y$-, and $z$-directions, whereas $u_{x}, u_{y}$, and $u_{z}$ are the corresponding flow velocity components. In Fig. 1(b), a cylindrical pipe is drafted where the cylindrical coordinate system has radial $r$-, azimuthal $\theta$-, and streamwise $z$-coordinates, and $u_{r}, u_{\theta}$, and $u_{z}$ are the corresponding flow velocity components. Let $H$ be the channel height and $D$ be the pipe diameter. The canonical channel flow considered here is periodic in both the $y$ - and the $z$-directions for the sake of simplicity, whereas the pipe flow is periodic solely in the $z$-direction. Here, only continuous pipe flow solutions are sought; therefore, $\partial u_{i} / \partial r \neq \pm \infty$ at $r=0$ for $i=r, \theta$, and $z$.

The characteristic length scale is $l_{c}=H / 2$ for the channel and $l_{c}=D / 2$ for the pipe. The characteristic velocity is the bulk velocity $\left(u_{c}=u_{b}\right)$ defined based on the steady state pressure-driven laminar solution corresponding to no-slip boundaries (i.e., Poiseuille flow ${ }^{33}$ ). The no-slip boundary conditions are $\boldsymbol{u}(H / 2, y, z, t)=\mathbf{0}$ and $\boldsymbol{u}(-H / 2$, $y, z, t)=\mathbf{0}$ in the channel and $\boldsymbol{u}(D / 2, \theta, z, t)=\mathbf{0}$ in the pipe. Using the nondimensional formulation, the streamwise pressure-gradientdriven laminar steady state solution is

$$
u_{z, \mathrm{Poi}}=3\left(1-x^{2}\right) / 2 \text {, and } p=-3 z / \operatorname{Re}+p_{0},
$$

for the channel flow, and

$$
u_{z, \text { Poi }}=2\left(1-r^{2}\right) \text {, and } p=-8 z / \operatorname{Re}+p_{0},
$$

for the pipe flow. Here, $p_{0}=0$ represents the zero level of the pressure. The bulk Reynolds numbers are $\operatorname{Re}=H u_{b} /(2 v)$ and $\operatorname{Re}=D u_{b} /(2 v)$ for the channel and the pipe flows, respectively.

For the analytical solutions, time and space-periodic in-plane wall motions are considered as boundary conditions. In order to ensure linearity (zero convective term), the wall-normal or radial velocity is set to zero. In Cartesian coordinate systems, this implies that $\partial p / \partial x=0$, whereas in cylindrical coordinates, $\partial p / \partial r=u_{\theta}^{2} / r$. Therefore, linear analytical solutions are available only if in-plane wall oscillations are prescribed. The considered wall motions imposed as boundary conditions (described in Secs. III and IV) introduce velocity fields which automatically satisfy the continuity Eq. (1) and describe stable, laminar flows. The corresponding channel and pipe flows are described by linear Partial Differential Equations (PDEs) which are the simplified version of Eq. (2), known as the unsteady Stokes equations. ${ }^{46}$ It is worth noticing though that here the Stokes equations are applied because of carefully selected boundary conditions and not because of the low Reynolds number assumption.

The governing equations in every case are Eqs. (1) and (2), and the solution procedure is as follows. First, the boundary conditions are set. Second, a hypothetical solution form is deduced from the boundary conditions and governing equations. The solution functions describe the space- and time-dependent velocity and potentially pressure fields within the domain of interest. Due to the confined domains and the time-periodic forcing, the velocity fields depend at least on time $(t)$ and the wall-normal $(x)$ or radial $(r)$ coordinate. Third, the hypothetical solution function is used to obtain the simplified (linear) governing equations. After separation of variables, the resulting linear PDEs are converted to Ordinary Differential Equations (ODEs) that are solved analytically. The coefficients in the solution functions can be found by matching the prescribed boundary conditions at the moving walls. Finally, the solutions of the simplified governing equations are analyzed to confirm that they satisfy the boundary conditions and the original governing equations.

\section{SOLUTIONS IN THE CHANNEL}

This section presents the parallel flow solutions in a Cartesian coordinate system. Only symmetric solutions are presented, bearing in mind that obtaining antisymmetric solutions from here is straightforward. The simplest flow problem involves uniform wall oscillations as boundary conditions

$$
u_{z, \text { uni }}(x= \pm 1, t)=\mathfrak{R}\left\{\sum_{j=1}^{N} A_{j} \mathrm{e}^{\mathrm{i} \omega_{j} t}\right\} .
$$

Here, $\mathfrak{R}$ indicates the real part, $N$ denotes the number of modes, whereas $A_{j}$ and $\omega_{j}$ are the complex amplitude and angular frequency of each mode, respectively. The second subscript is purely used to distinguish between different cases [e.g., in Eq. (5) "uni" stands for uniform]. The corresponding hypothetical solution form $u_{z \text {,uni }}(x, t)$ involves a single streamwise velocity component and is deduced from Eqs. (5) and (2). Substituting $u_{z \text {,uni }}(x, t)$ into Eqs. (1) and (2) highlights that the governing equations take the form of a simple diffusion equation

$$
\frac{\partial u_{z}}{\partial t}-\frac{1}{\operatorname{Re}} \frac{\partial^{2} u_{z}}{\partial x^{2}}=0
$$

The solution function with the boundary conditions (5) is

$$
u_{z, \text { uni }}=\Re\left\{\sum_{j=1}^{N} A_{j} \frac{\cos \left(\sqrt{-\mathrm{i}} \sqrt{\omega_{j} \operatorname{Re} x}\right)}{\cos \left(\sqrt{-\mathrm{i}} \sqrt{\omega_{j} \operatorname{Re}}\right)} \mathrm{e}^{\mathrm{i} \omega_{j} t}\right\} .
$$

Substituting $x= \pm 1$ into Eq. (7) shows that the oscillating boundary conditions governed by Eq. (5) are satisfied. Furthermore, substituting Eq. (7) into Eqs. (1) and (2) shows that this solution (and $u_{x}=u_{y}$ $=p=0$ ) satisfies the complete set of the original governing equations in addition to the simplified Eq. (6). In the following, the same steps are applied for different boundary conditions.

Next, consider a channel flow solution introduced by wall oscillations in traveling wave (tw) form (boundary condition)

$$
u_{z, \text { tw }}(x= \pm 1, y, t)=\Re\left\{\sum_{j=1}^{N} A_{j} \mathrm{e}^{\mathrm{i} k_{j} y-\mathrm{i} k_{j} c_{j} t}\right\} .
$$

The angular wave number and phase velocity of each mode are $k_{j}$ and $c_{j}$, respectively. The corresponding solution form $u_{z, \mathrm{tw}}(x$, $y, t)$ depends on time and two spatial coordinates, but nevertheless has only one, streamwise velocity component. The simplified momentum equation is given by

$$
\frac{\partial u_{z}}{\partial t}-\frac{1}{\operatorname{Re}}\left[\frac{\partial^{2} u_{z}}{\partial x^{2}}+\frac{\partial^{2} u_{z}}{\partial y^{2}}\right]=0
$$

and the solution is

$$
u_{z, \mathrm{tw}}=\Re\left\{\sum_{j=1}^{N} A_{j} \frac{\cos \left(\sqrt{\mathrm{i}} \sqrt{\mathrm{i} k_{j}^{2}+k_{j} c_{j} \operatorname{Re} x}\right)}{\cos \left(\sqrt{\mathrm{i}} \sqrt{\mathrm{i} k_{j}^{2}+k_{j} c_{j} \operatorname{Re}}\right)} \mathrm{e}^{\mathrm{i} k_{j} y-\mathrm{i} k_{j} c_{j} t}\right\} .
$$


The product of the angular wave number and phase velocity can be introduced as the angular frequency of the wall oscillation $\left(\omega_{j}=k_{j} c_{j}\right)$.

Modes with positive phase velocity $\left(c_{j}>0\right)$ travel in the positive spanwise direction and vice versa. Traveling wave mode-pairs with equivalent amplitude but with opposite phase velocity leads to standing wave (sw) wall oscillations

$$
\begin{aligned}
u_{z, \mathrm{sw}}(x= \pm 1, y, t) & =\Re\left\{\sum_{j=1}^{N}\left(\frac{1}{2} A_{j} \mathrm{e}^{\mathrm{i} k_{j} y-\mathrm{i} k_{j} c_{j} t}\right)+\sum_{j=1}^{N}\left(\frac{1}{2} A_{j} \mathrm{e}^{\mathrm{i} k_{j} y+\mathrm{i} k_{j} c_{j} t}\right)\right\} \\
& =\Re\left\{\sum_{j=1}^{N} A_{j} \mathrm{e}^{\mathrm{i} k_{j} y} \cos \left(\omega_{j} t\right)\right\} .
\end{aligned}
$$

Hence, linear analytical solutions enable to obtain channel flow solutions driven by traveling as well as standing wave wall oscillations.

If only spanwise wall oscillations are applied, then the above procedure leads to spanwise velocity fields denoted by $u_{y \text {, uni }}(x, t)$ and $u_{y, \mathrm{tw}}(x, z, t)$. These solutions are of similar form to (7) and (10) ( $y$ needs to be replaced by $z$ in the formulas). The $u_{z, \text { uni }}, u_{z, \mathrm{tw}}$, and $u_{y}$,uni solutions can be superimposed on any linear flow solution which is driven by the streamwise pressure gradient $(\partial p / \partial z)$ or steady wall motion. Furthermore, the $u_{z \text {,uni }}$ and $u_{y \text {, uni }}$ solutions can be combined with each other and flows driven by the pressure gradient or steady wall motion, for instance, Couette, Poiseuille $\left(u_{z, \mathrm{Poi}}\right)$, and Womersley flow solutions.

\section{SOLUTIONS IN THE PIPE}

For the pipe, a parallel flow solution is obtained by considering a uniform streamwise wall oscillation as the boundary condition

$$
u_{z, \text { uni }}(r=1, t)=\Re\left\{\sum_{j=1}^{N} A_{j} \mathrm{e}^{\mathrm{i} \omega_{j} t}\right\} .
$$

This forcing results in a single streamwise velocity component as functions of the radial coordinate and time. After substituting $u_{z \text {,uni }}(r, t)$ into Eq. (2), a diffusion equation in cylindrical coordinates can be written as

$$
\frac{\partial u_{z}}{\partial t}-\frac{1}{\operatorname{Re}}\left[\frac{1}{r} \frac{\partial u_{z}}{\partial r}+\frac{\partial^{2} u_{z}}{\partial r^{2}}\right]=0 .
$$

The particular solution satisfying the imposed boundary conditions reads as

$$
u_{z, \text { uni }}=\Re\left\{\sum_{j=1}^{N} A_{j} \frac{J_{0}\left(\sqrt{-\mathrm{i} \omega_{j} \operatorname{Re} r}\right)}{J_{0}\left(\sqrt{-\mathrm{i} \omega_{j} \operatorname{Re}}\right)} \mathrm{e}^{\mathrm{i} \omega_{j} t}\right\} .
$$

Here, $J_{0}$ is the Bessel function of the first kind with order zero.

Parallel pipe flow can also be introduced by applying streamwise wall excitation taking traveling wave forms so that

$$
u_{z, \mathrm{tw}}(r=1, \theta, t)=\Re\left\{\sum_{j=1}^{N} A_{j} \mathrm{e}^{\mathrm{i} k_{j} \theta-\mathrm{i} k_{j} c_{j} t}\right\} .
$$

Here, the angular wave number is an integer so that $k_{j}=j$. The simplified governing equation reads

$$
\frac{\partial u_{z}}{\partial t}-\frac{1}{\operatorname{Re}}\left[\frac{1}{r} \frac{\partial u_{z}}{\partial r}+\frac{\partial^{2} u_{z}}{\partial r^{2}}+\frac{1}{r^{2}} \frac{\partial^{2} u_{z}}{\partial \theta^{2}}\right]=0 .
$$

The corresponding continuous particular solutions $u_{z, \mathrm{tw}}(r, \theta, t)$ is defined by

$$
u_{z, \mathrm{tw}}=\Re\left\{\sum_{j=1}^{N} A_{j} \frac{I_{j}\left(-\mathrm{i} \sqrt{\mathrm{i} k_{j} c_{j} \operatorname{Re} r}\right)}{I_{j}\left(-\mathrm{i} \sqrt{\mathrm{i} k_{j} c_{j} \operatorname{Re}}\right)} \mathrm{e}^{\mathrm{i} k_{j} \theta-\mathrm{i} k_{j} c_{j} t}\right\},
$$

where $I_{j}$ is the modified Bessel function of the first kind with order $j$. The traveling wave solutions naturally include standing wave solutions $\left(u_{z, \mathrm{sw}}\right)$ similarly to channel flows. Furthermore, the $u_{z, \text { uni }}, u_{z \text {,sw }}$, and $u_{z \text {,tw }}$ solutions in the pipe are analogous to the $u_{z \text {,uni }}, u_{z \text {,sw }}$, and $u_{z, \mathrm{tw}}$ solutions in the rectangular channel.

Following the same logic, concentric flow solutions can be obtained for azimuthal wall velocity oscillations described by

$$
u_{\theta, \text { uni }}(r=1, t)=\mathfrak{R}\left\{\sum_{j=1}^{N} A_{j} \mathrm{e}^{\mathrm{i} \omega_{j} t}\right\} .
$$

With $u_{\theta \text {,uni }}(r, t)$ solution form, the simplified governing equations are

$$
\left.\begin{array}{l}
\frac{u_{\theta}}{\partial t}-\frac{1}{\operatorname{Re}}\left[\frac{1}{r} \frac{\partial u_{\theta}}{\partial r}+\frac{\partial^{2} u_{\theta}}{\partial r^{2}}-\frac{u_{\theta}}{r^{2}}\right]=0 \\
\frac{u_{\theta}^{2}}{r}-\frac{\partial p}{\partial r}=0
\end{array}\right\}
$$

The continuous particular solutions of the velocity and the pressure fields are

$$
\begin{aligned}
& u_{\theta, \text { uni }}=\Re\left\{\sum_{j=1}^{N} A_{j} \frac{J_{1}\left(\sqrt{-\mathrm{i} \omega_{j} \operatorname{Re} r}\right)}{J_{1}\left(\sqrt{-\mathrm{i} \omega_{j} \mathrm{Re}}\right)} \mathrm{e}^{\mathrm{i} \omega_{j} t}\right\}, \text { and } \\
& p_{\text {uni }}=\int \frac{u_{\theta, \text { uni }}^{2}(r, t)}{r} \mathrm{~d} r .
\end{aligned}
$$

The solution $u_{\theta \text {,uni }}$ is analogous to $u_{y \text {,uni }}$ in the rectangular channel.

Whereas the aforementioned diffusion equations can be found in the literature related to heat conduction (e.g., Ref. 47), Eq. (19) and its corresponding velocity field appear only to have been documented by Berker, ${ }^{40,41}$ who eliminated the pressure gradient term as a by-product of the vorticity-stream function formulation. The pressure field is more complicated than the velocity field and goes through more rapid changes as a function of time because of the quadratic velocity term in the argument of the integral. It is interesting to see that the radial pressure gradient is necessary to balance the rotation of the fluid rings and prevent the growth of radial acceleration. This flow is reminiscent of rotating boundary layer flows, ${ }^{48}$ such as the Ekman layer ${ }^{49-51}$ characterized by the balance of pressure gradient, viscous, and Coriolis forces.

$\mathrm{Wang}^{38}$ listed the governing equations for concentric flows as

$$
\left.\begin{array}{l}
\frac{u_{\theta}^{2}}{r}-\frac{\partial p}{\partial r}=0 \\
\frac{u_{\theta}}{\partial t}-\frac{1}{\operatorname{Re}}\left[\frac{1}{r} \frac{\partial u_{\theta}}{\partial r}+\frac{\partial^{2} u_{\theta}}{\partial r^{2}}-\frac{u_{\theta}}{r^{2}}+\frac{\partial^{2} u_{\theta}}{\partial z^{2}}\right]=0
\end{array}\right\}
$$


The equations imply that linear azimuthal velocity solutions can depend not only on $r$ and $t$ but also on $z$. The solution $u_{\theta}(r, z, t)$ in the pipe would be analogous to the $u_{y, \mathrm{sw}}$ or $u_{y, \text { tw }}$ channel flow solution, and this solution form includes streamwise-traveling azimuthal wall oscillations, also investigated experimentally. ${ }^{16}$ To gain further understanding of this scenario, the Navier-Stokes momentum equations in the cylindrical coordinate system are expanded considering an initial velocity field $\left[u_{r}=0 ; u_{\theta}(r, z) ; u_{z}=0\right]$,

$$
\begin{gathered}
-\frac{u_{\theta}^{2}}{r}=-\frac{\partial p}{\partial r} \\
\frac{\partial u_{\theta}}{\partial t}=\frac{1}{\operatorname{Re}}\left[\frac{1}{r} \frac{\partial}{\partial r}\left(r \frac{\partial u_{\theta}}{\partial r}\right)-\frac{u_{\theta}}{r^{2}}+\frac{\partial^{2} u_{\theta}}{\partial z^{2}}\right] \\
\frac{\partial u_{z}}{\partial t}=-\frac{\partial p}{\partial z} .
\end{gathered}
$$

Letting the azimuthal velocity $u_{\theta}(r, z, t)$ depend on both the axial and the radial coordinates implies a pressure field $p(r, z, t)$ depending on the axial and the radial coordinates, as suggested by Eq. (22). The resulting pressure field leads to a nonzero right-hand side in Eq. (24) and generates axial velocity. The emerging axial velocity depends on $z$ as dictated by the axial pressure gradient. Because of the continuity equation

$$
\frac{1}{r} \frac{\partial\left(r u_{r}\right)}{\partial r}+\frac{1}{r} \frac{\partial u_{\theta}}{\partial \theta}+\frac{\partial u_{z}}{\partial z}=0
$$

the axial velocity introduces radial velocity and/or azimuthal dependence of the azimuthal velocity and hence lead to nonlinearity. Examining Eqs. (21)-(25) in more detail, it appears that a unidirectional velocity field in the form of $u_{\theta}(r, z, t)$ contradicts the governing equations (1) and (2). For this reason, linear solutions of the azimuthal velocity field exist only for $u_{\theta}(r, t)$ which prevents the appearance of nonlinearity. In short, a linear solution does not exist in the $u_{\theta}(r, z, t)$ form and so concentric flows are described by Eq. (19) instead of (21).

Superimposition is also possible for pipe flow solutions. Solutions $u_{z \text {,uni }}, u_{z, \mathrm{sw}}, u_{z, \mathrm{tw}}, u_{\theta \text {,uni }}$ can be superimposed on other axial pressure gradient driven laminar flows. To achieve helical flow solutions, it is possible to combine $u_{z \text {,uni }}(r, t)$ and $u_{\theta \text {,uni }}(r, t)$. In this case, we need to solve the equation set including Eqs. (13) and (19). The solutions are then given by Eqs. (14) and (20) where the oscillations of axial and azimuthal velocity components are accompanied by changes in the radial pressure gradient.

\section{RESULTS AND DISCUSSION}

The exact analytical solutions presented in Sec. II describe laminar flows driven by uniform and traveling wave wall oscillations. In this section, first uniform wall oscillations are analyzed, and then traveling wave wall oscillations are examined. The analytical solutions allow the description of arbitrary space- and time-periodic continuous wall motions. Complex wall motions can be defined by the corresponding Fourier modes so that the solution field of each mode can be superimposed. For simplicity, only monoharmonic oscillations are considered, bearing in mind that extending the following analysis to multiple Fourier modes is straightforward. For visualization purposes, the oscillating velocity amplitude of the controlled walls is set to unity and $\operatorname{Re}=1000$.

\section{A. Uniform wall oscillations}

Flows driven by uniform streamwise or spanwise/azimuthal wall oscillations depend on the wall-normal/radial coordinate and
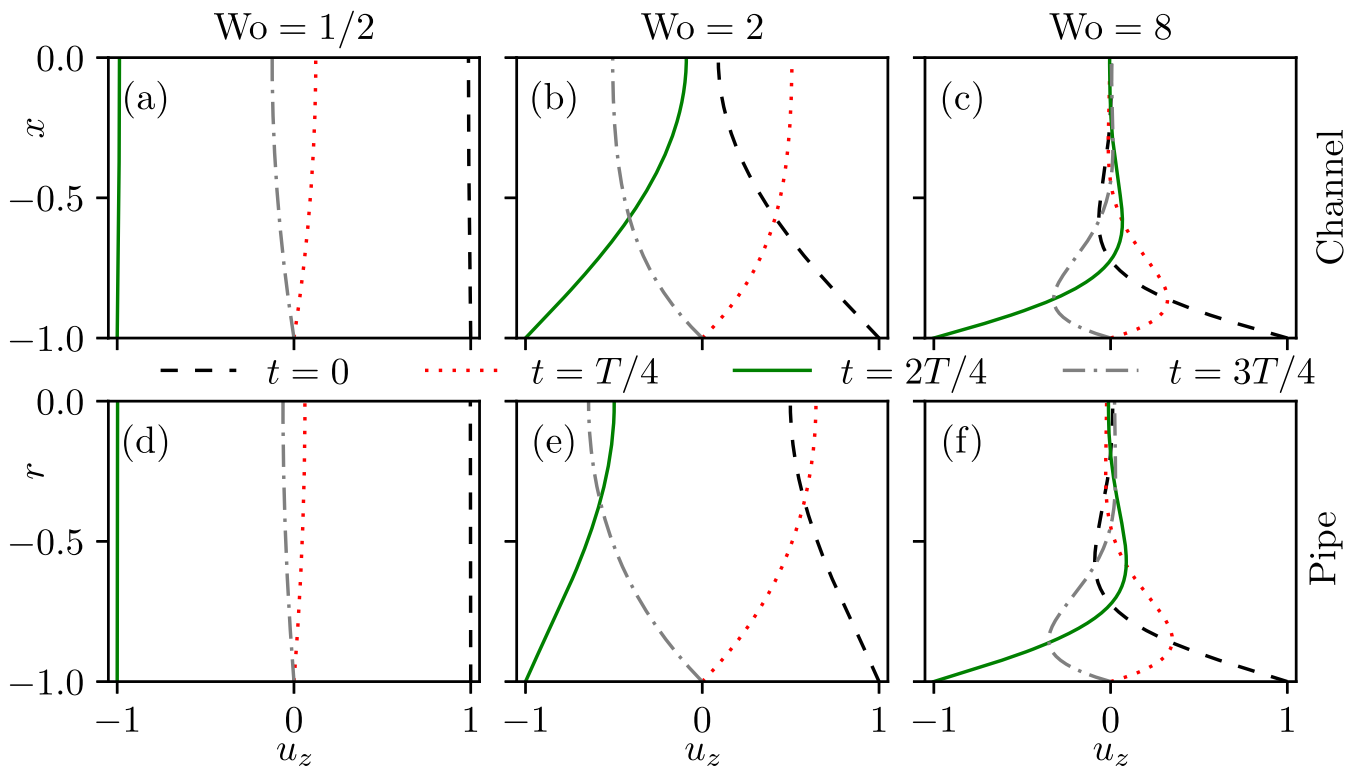

FIG. 2. Velocity profiles in the channel (a)-(c) and pipe flows (d)-(f) driven by uniform streamwise wall oscillations as functions of wall-normal $(x)$ or radial $(r)$ coordinates Various time-instances and Womersley numbers (Wo) are presented. Only the lower half of the channel is shown because the solution functions are symmetric. 
time. Instead of the bulk Reynolds number (Re), these flows can be characterized by the Womersley number (Wo) describing the ratio of pulsatile inertial and viscous forces as suggested by nondimensionalization. ${ }^{45}$ The Womersley number is defined as

$$
\mathrm{Wo}=\sqrt{2 \pi \mathrm{ReSt}},
$$

where

$$
\mathrm{St}=\left(\omega l_{c}\right) /\left(2 u_{b} \pi\right)
$$

is the Strouhal number (for the channel $l_{c}=H / 2$ and for the pipe $l_{c}=D / 2$.)

Figure 2 depicts the oscillating velocity profiles in channel and pipe flows driven by streamwise wall oscillations incorporating a single mode. The flow fields are reminiscent of the Stokes boundary layer $^{33}$ and pulsatile Womersley flow. ${ }^{45}$ With an increasing Womersley number, the wall oscillations energize a thinning fluid layer [compare Figs. 2(a)-2(c) and Figs. 2(d)-2(f)]. Below a certain Womersley number $\left(\right.$ Wo $_{\approx} 0.2$ ), the fluid motion throughout the channel/pipe is uniform and well approximated by the wall motion as visualized in Figs. 2(a) and 2(d).

Velocity profiles in the pipe flow corresponding to monoharmonic azimuthal wall oscillations are displayed in Figs. 3(a)-3(c).
Similarly to flows driven by uniform axial wall oscillations, the energized fluid layer becomes thinner with increasing Womersley number as the inertial forces start to dominate. However, due to the axisymmetric flow field, the fluid velocity in the centerline of the pipe remains zero. Another major difference compared to axial flows is that the azimuthal flow field is balanced by a radial pressure gradient. The pipe flow driven by wall oscillations is a special case of linear analytical solutions, when two out of three components of the momentum equations are satisfied. The pressure distribution is shown in Figs. 3(d)-3(f). Observing the history of the wall pressure and wall velocity reveals that, in this monoharmonic scenario, the frequency of pressure oscillations is double of the velocity oscillations [Figs. 3(g)-3(i)]. The pressure field is governed by Eq. (20), where a quadratic relationship is highlighted between the velocity and the pressure fields. Because of this connection, the pressure of concentric flows always has modes which oscillate faster than the velocity field.

Channel and pipe flows driven by uniform wall oscillations are reminiscent of the Stokes boundary layer corresponding to oscillating flat plates. In fact, the Stokes boundary layer has been used to approximate the phase-averaged oscillations caused by uniform wall motions in turbulent channel flows. ${ }^{12}$

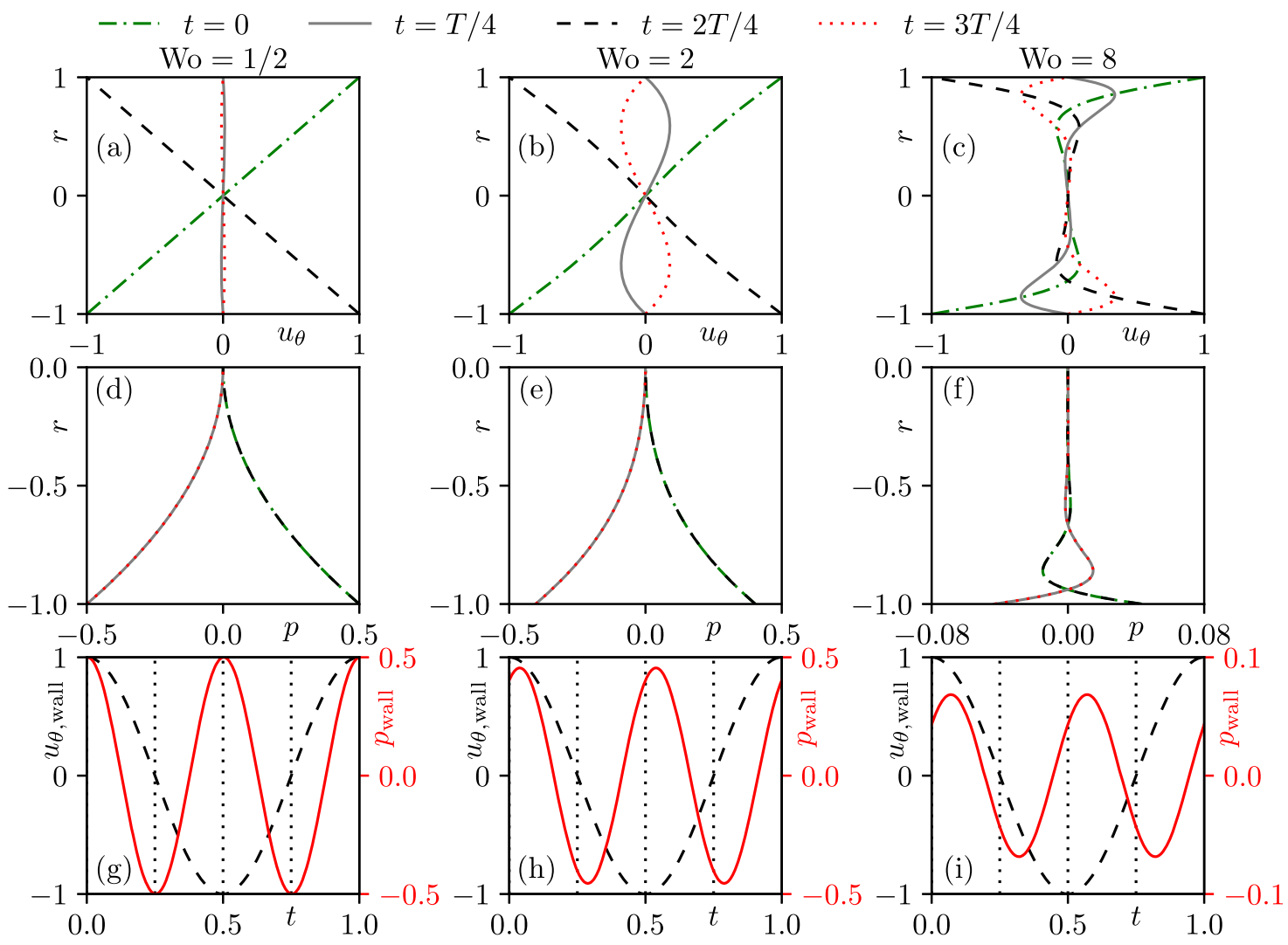

FIG. 3. Velocity profiles (a)-(c) and pressure distribution (d)-(f) in the pipe driven by uniform azimuthal wall oscillations as functions of radial coordinate $(r)$. Various timeinstances and Womersley numbers (Wo) are presented. In (d)-(f), only negative radial coordinates are depicted because the pressure functions are axisymmetric. The time history of the wall velocity $\left(u_{\theta, \text { wall }}\right)$ and wall pressure $\left(p_{\text {wall }}\right)$ is depicted in $(\mathrm{g})-(\mathrm{i})$, where the dotted lines correspond to the time instants shown in $(\mathrm{a})-(\mathrm{f})$. 

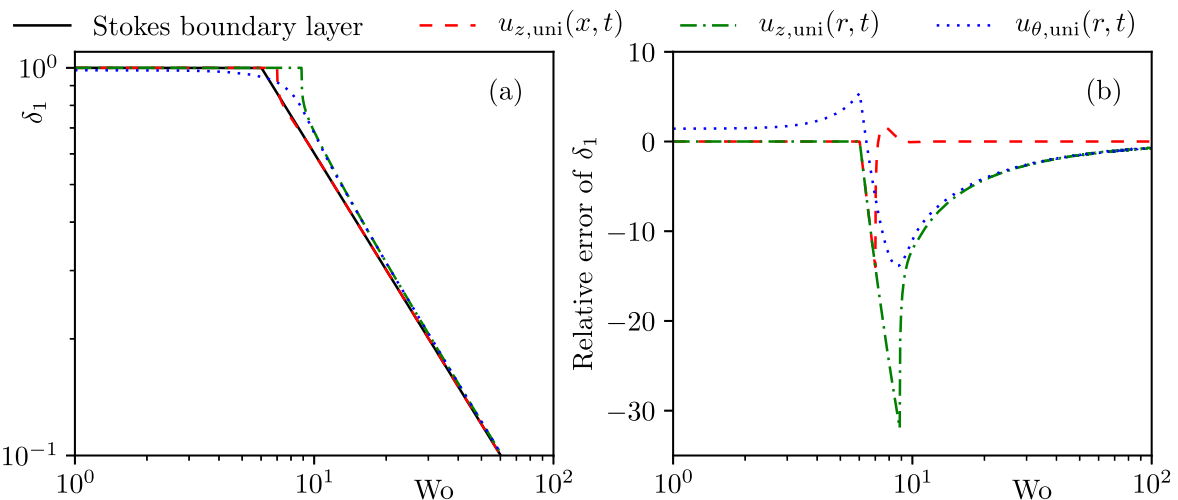

FIG. 4. Oscillating boundary layer thickness corresponding to analytical solutions of flat plate, parallel channel $\left[u_{z, \text { uni }}(x, t)\right]$, and parallel $\left[u_{z, \text { uni }}(r, t)\right]$ and concentric $\left[u_{\theta}\right.$,uni $\left.(r, t)\right]$ pipe flows (a). Relative error of boundary layer thickness estimation based on the oscillating flat plate flow (b).
However, the Stokes boundary layer cannot take into account that channel/pipe flows are bounded in the wall-normal/radial direction. This difference in the boundary conditions starts to play a role as the oscillating boundary layer approaches the symmetry plane of the channel or the centerline of the pipe.

In order to quantify the difference between oscillating flat plate, channel, and pipe flows, the oscillating boundary layer thickness is introduced. For parallel flows, for instance, the oscillating boundary layer thickness $\left(\delta_{1}\right)$ is computed from

$$
\sqrt{\frac{\int_{0}^{T}\left[u_{z, \text { uni }}\left(-1+\delta_{1}, t\right)\right]^{2} \mathrm{~d} t}{\int_{0}^{T}\left[u_{z, \text { uni }}(-1, t)\right]^{2} \mathrm{~d} t}}=\frac{u_{z, \text { uni }}^{\mathrm{rms}}\left(-1+\delta_{1}, t\right)}{u_{z, \text { uni }}^{\mathrm{rms}}(-1, t)}=\frac{1}{100}
$$

Here, $u_{z \text {,uni }}^{\mathrm{rms}}\left(x_{0}, t\right)$ denotes the root-mean-square of the velocity oscillation at a given location $\left(x_{0}\right) . T=2 \pi / \omega$ is the monoharmonic oscillation period. According to Eq. (28), $\delta_{1}$ shows how far from the wall fluid oscillation intensity reaches $1 \%$ of the wall oscillation intensity. In the case of concentric flows, $\delta_{1}$ is defined following the same principle.

Figure 4(a) displays the boundary layer thickness as a function of the Womersley number. As Wo $\rightarrow \infty$ and Wo $\rightarrow 0$, the oscillating flat plate boundary layer gives the same results as the channel and pipe flow solutions. It is also worth noticing that as $\mathrm{Wo} \rightarrow \infty$, the decay of the boundary layer thickness is well approximated by a power function. As expected, the flat plate approximation differs significantly from the oscillating channel and pipe flow solutions around $\mathrm{Wo}=8$ where the boundary layer reaches the symmetry plane or the center line. The relative error of the boundary layer thickness estimation based on the oscillating flat plate flow is shown in Fig. 4(b). Considering the pipe flows, the error of flat plate approximation can be as high as $30 \%$. It is not surprising that the oscillating flat plate approximates better the flow conditions in the channel than in the pipe.

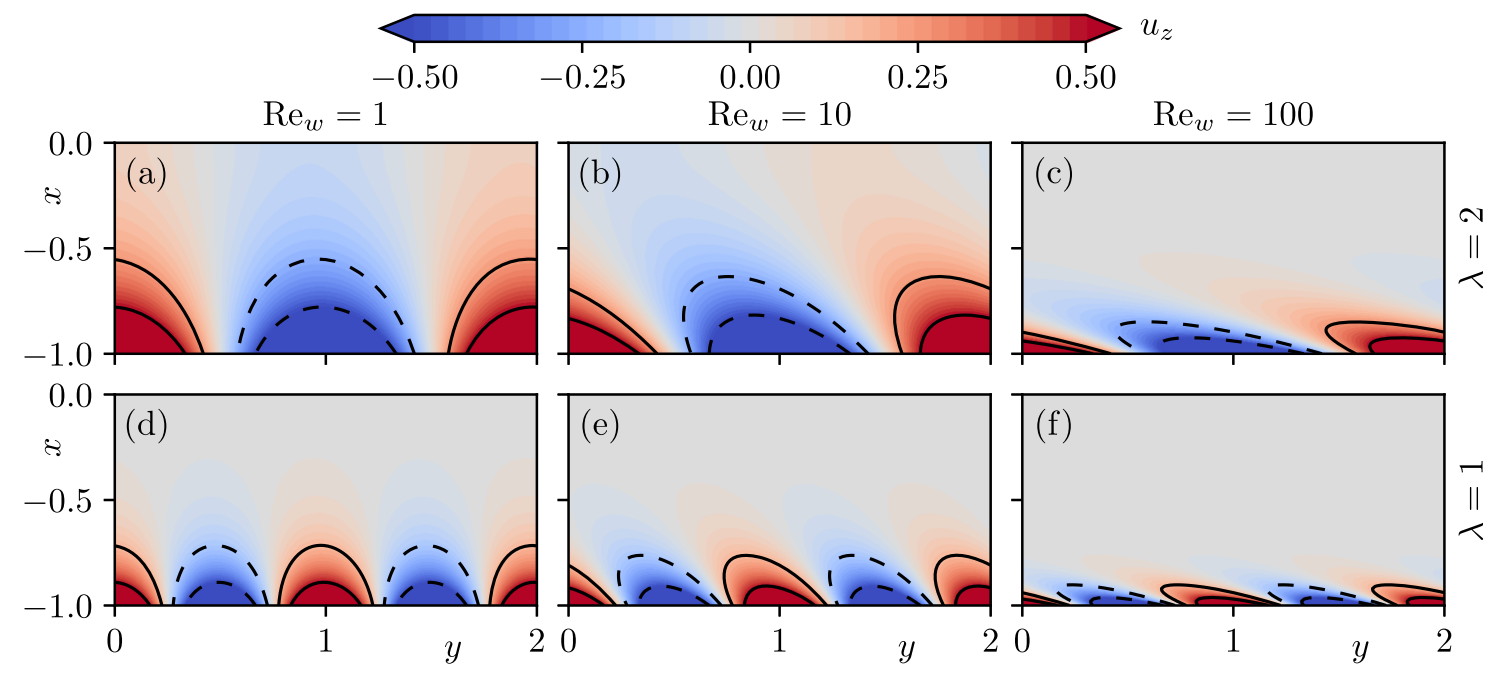

FIG. 5. Contour plots of traveling wave solutions of the streamwise velocity with a positive spanwise phase velocity as functions of the spanwise $(y)$ and wall-normal $(x)$ coordinates. Solid and dashed lines symbolize positive and negative contours, respectively, at $u_{z}= \pm 0.5$ and \pm 0.25 . The (a)-(c) and (d)-(f) subfigures illustrate the effect of increasing wave Reynolds number and peripheral wave length. 

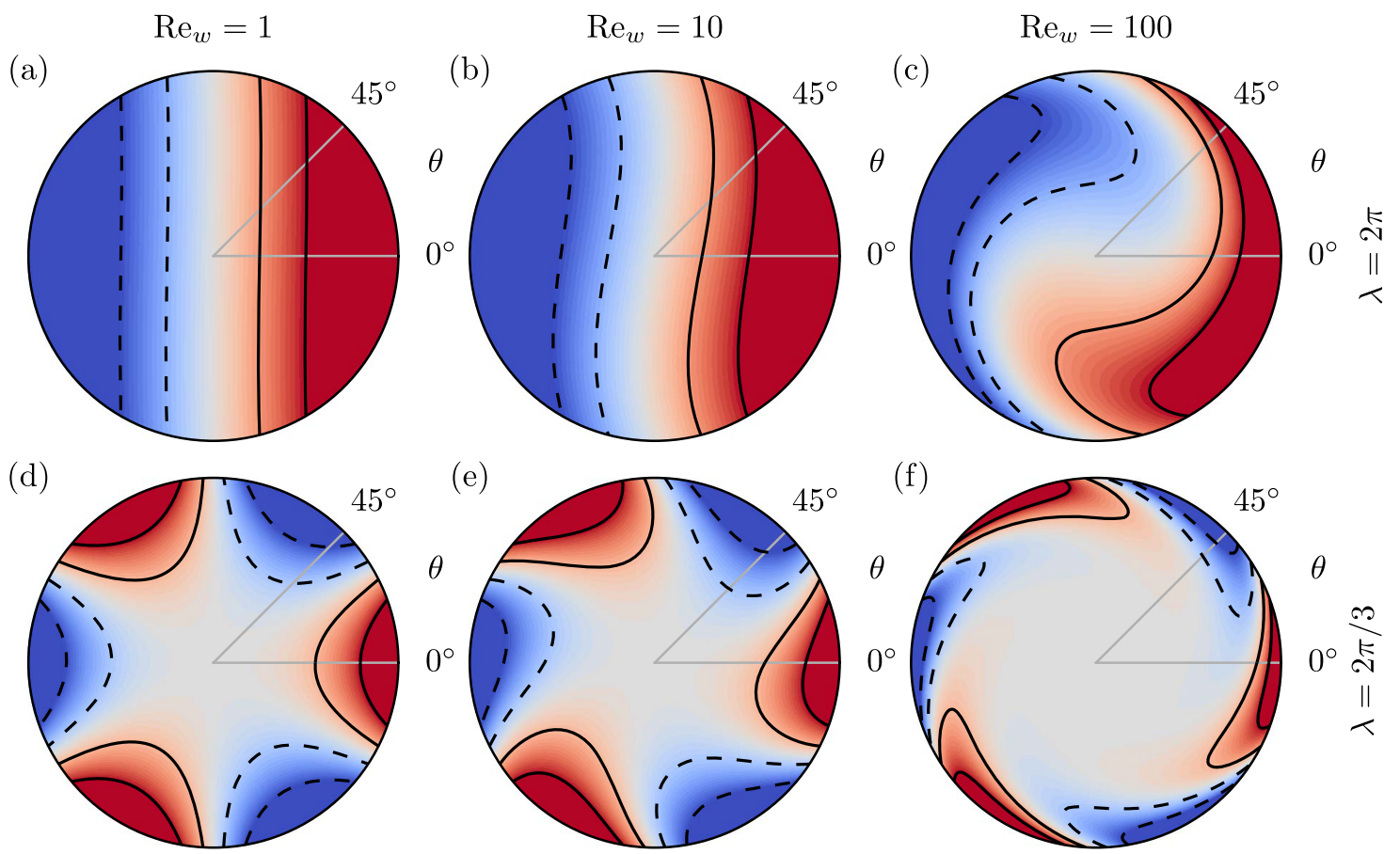

FIG. 6. Contour plots of traveling wave solutions of the streamwise velocity with a positive azimuthal phase velocity as functions of the azimuthal $(\theta)$ and radial coordinates Solid and dashed lines symbolize positive and negative contours, respectively, at $u_{z}= \pm 0.5$ and \pm 0.25 . The color map is the same as in Fig. 5 . The (a)-(c) and (d)-(f) subfigures illustrate the effect of increasing wave Reynolds number and peripheral wave length.

\section{B. Traveling wave wall oscillations}

In the case of the channel and pipe flows driven by traveling wave wall oscillations, the Stokes boundary layer is not suitable to approximate the velocity fields because it does not contain information about the spatial scale of the wall motions. For the same reason, the Womersley number is not sufficient any more to characterize the flow field. The question may arise: what is the similarity criterion of these flows?

Dimensional analysis of the flows driven by traveling wave wall oscillations suggests that new characteristic scales should be selected based on the properties of the wall motions. Using the peripheral wave length $(\lambda=2 \pi / k)$ as characteristic length scale $\left(l_{c}^{w}=\lambda\right)$ and the oscillation period as characteristic time scale $\left(t_{c}^{w}=T=2 \pi / \omega\right)$ leads
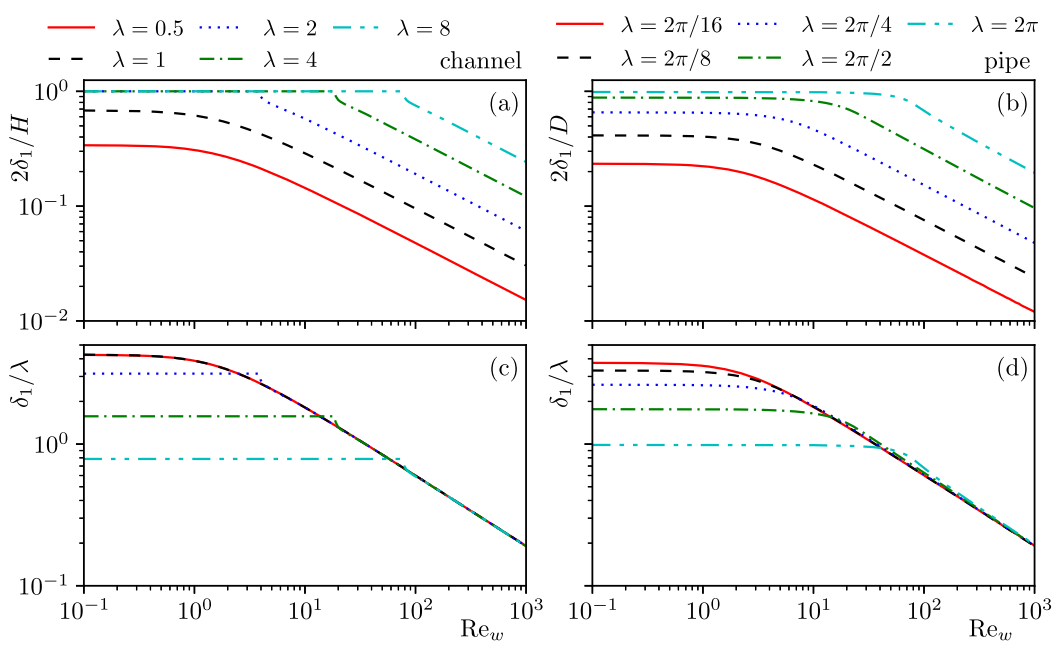

FIG. 7. Oscillating boundary layer thickness as a function of the wave Reynolds number corresponding to analytical solutions comprising traveling wave wall motions in channel (a)-(c) and pipe (b)-(d) flows. The (a)-(c) and (b)-(d) pairs visualize the same data. In subfigures (a) and (b), $\delta_{1}$ is nondimensionalized with the channel half-height $(H / 2)$ and the radius ( $D / 2)$, whereas in (c) and (d), the peripheral wave length $\lambda$ is used as reference length scale. 
to a new dimensionless group. The resulting characteristic velocity scale is the phase velocity $u_{c}^{w}=\lambda \omega /(2 \pi)=c$, and the wave Reynolds number can be defined as

$$
\operatorname{Re}_{w}=\frac{\lambda^{2} \omega}{2 \pi v} .
$$

Figures 5 and 6 visualize how the quasi two-dimensional velocity fields induced by the traveling wave wall oscillations depend on the wave Reynolds number. The high and low momentum fluid regions introduced by the wall oscillations become skewed and the energized fluid layer thinner with increasing $\mathrm{Re}_{w}$. With waves traveling in the positive spanwise/azimuthal direction, the velocity field is skewed toward the negative spanwise/azimuthal direction and vice versa. Figures 5 and 6 also demonstrate that decreasing peripheral wave length implies decreasing boundary layer thickness. This observation draws attention to the ratio between the peripheral wave length and the channel height or pipe diameter and their connection to the oscillating boundary layer thickness.

Figures 7(a) and 7(b) depict the oscillating boundary layer thickness in the channel and the pipe as a function of the wave length and wave Reynolds number. As $\operatorname{Re}_{w} \rightarrow 0$, the boundary layer thickness reaches a plateau in every case and does not increase further. In the case of the channel, above a certain peripheral wave length $(2 \lambda / H \approx 1.27)$, as $\operatorname{Re}_{w} \rightarrow 0$, the boundary layer thickness becomes equal to the channel half-height. On the contrary, the boundary layer thickness in the pipe never reaches the radius but converges to it asymptotically. As $\operatorname{Re}_{w} \rightarrow \infty$, the boundary layer thickness decays following a power function, similarly to the boundary layer above uniform wall oscillations (see Fig. 4). Plotting the boundary layer thickness nondimensionalized with the wave length of the traveling wave wall oscillations, as shown in Figs. 7(c) and 7(d), draws attention to other trends. In the channel, the behavior of the oscillating boundary layer is independent from the wave length of the traveling waves as long as the oscillating boundary layer does not reach the centerline. This statement is supported by the overlapping line sections in Figs. 7(c) and 7(d).

\section{CONCLUSIONS}

This study has investigated channel and pipe flows driven by in-plane uniform and traveling wave wall motions in channel and pipe flows. A comprehensive collection of exact linear analytical solutions has been presented comprising seven analytical solutions summarized in Table I. The corresponding flow fields describe one- and two-dimensional parallel and concentric laminar flows. Even though the analysis has been restricted to monoharmonic scenarios, solutions with multiple modes can be superimposed straightforwardly.

The Womersley number has been identified as the similarity parameter of channel and pipe flows driven by uniform wall oscillations. A new dimensionless group, namely, the wave Reynolds number, has been introduced to characterize confined flows driven by traveling wave wall oscillations. Instantaneous flow features has been characterized as functions of these dimensionless numbers. In addition, the oscillating boundary layer thickness has been analyzed, an important parameter to narrow the region of interest in the parameter space of turbulent flow controls. The analytical solutions open the way to investigations beyond monoharmonic wall oscillations.

The presented solutions could be also used for validation and verification purposes. The solution describing concentric pipe flows driven by uniform wall oscillations seems particularly useful to validate the pressure-velocity coupling of incompressible Navier-Stokes solvers $^{52-55}$ and quantify the temporal evolution of the numerical uncertainty. The unidirectional solutions can be extended to flows with hollowed cross sections and combined with related laminar flows, such as the Taylor-Couette flow. ${ }^{56}$ Periodically driven TaylorCouette turbulence ${ }^{57}$ has received attention recently because accelerating turbulent flows are in the focus of interes. ${ }^{58,59}$ Linear stability analysis $^{42-44}$ could be utilized to exploit how these solutions influence the natural laminar-turbulent transition of channel and pipe flows. Furthermore, some of the analytical solutions might be realized experimentally using existing techniques ${ }^{9,10,16-18}$ and utilized to calibrate pressure and velocity sensors, and actuators.

The author hopes that the results will further the development of active flow controls with multiple modes for high Reynolds number flows and help the design of microfluidic systems and biosensors.

\section{ACKNOWLEDGMENTS}

T. I. Józsa is grateful to Professor A. G. L. Borthwick of University of Edinburgh for useful discussions and suggestions regarding

TABLE I. Summary of the laminar channel and pipe flows driven by in-plane wall motions.

\begin{tabular}{|c|c|c|c|c|}
\hline Domain & Flow field & Flow direction & Wall motion & Solution function \\
\hline \multirow[t]{2}{*}{ Channel } & \multirow{2}{*}{ Parallel } & $\begin{array}{l}\text { Streamwise } \\
\text { Spanwise }\end{array}$ & Uniform & $\begin{array}{c}u_{z, \text { uni }}(x, t), \text { Eq. } \\
u_{y, \text { uni }}(x, t)\end{array}$ \\
\hline & & $\begin{array}{l}\text { Streamwise } \\
\text { Spanwise }\end{array}$ & Traveling wave & $\begin{array}{c}u_{z, \mathrm{tw}}(x, y, t), \text { Eq. } \\
u_{y, \mathrm{tw}}(x, z, t)\end{array}$ \\
\hline \multirow[t]{2}{*}{ Pipe } & Parallel & $\begin{array}{l}\text { Streamwise } \\
\text { Streamwise }\end{array}$ & $\begin{array}{c}\text { Uniform } \\
\text { Traveling wave }\end{array}$ & $\begin{array}{c}u_{z, \text { uni }}(r, t), \text { Eq. } \\
u_{z \text {,uni }}(r, \theta, t), \text { Eq. }\end{array}$ \\
\hline & Concentric & Azimuthal & Uniform & $u_{\theta, \text { uni }}(r, t)$, Eq. $(20)$ \\
\hline
\end{tabular}


the analytical solutions and the structure of this paper. In addition, T. I. Józsa wishes to thank K. J. Grant of University of Edinburgh for proofreading the text. The presented research has not received dedicated funding but has partially taken place as part of a project. As such, the author would like to acknowledge financial support from the University of Edinburgh, AkzoNobel's Marine Coatings business (International Paint Ltd.), and the Energy Technology Partnership (No. ETP106).

\section{REFERENCES}

${ }^{1}$ K. Länge, B. E. Rapp, and M. Rapp, "Surface acoustic wave biosensors: A review," Anal. Bioanal. Chem. 391, 1509-1519 (2008).

${ }^{2}$ C.-Y. Lee, C.-L. Chang, Y.-N. Wang, and L.-M. Fu, "Microfluidic mixing: A review," Int. J. Mol. Sci. 12, 3263-3287 (2011).

${ }^{3}$ L. N. Cattafesta III and M. Sheplak, "Actuators for active flow control," Annu. Rev. Fluid Mech. 43, 247-272 (2011).

${ }^{4}$ P. Ricco and P. D. Hicks, "Streamwise-travelling viscous waves in channel flows," J. Eng. Math. 111, 23-49 (2018).

5. Jiménez, "Near-wall turbulence," Phys. Fluids 25, 101302 (2013).

${ }^{6}$ International Energy Agency, Key World Energy Statistics (International Energy Agency, 2017)

${ }^{7} \mathrm{M}$. Gad-el-Hak, "Interactive control of turbulent boundary layers-A futuristic overview," AIAA J. 32, 1753-1765 (1994).

${ }^{8}$ W. J. Jung, N. Mangiavacchi, and R. Akhavan, "Suppression of turbulence in wall-bounded flows by high-frequency spanwise oscillations," Phys. Fluids 4, 1605-1607 (1992).

${ }^{9}$ F. Laadhari, L. Skandaji, and R. Morel, "Turbulence reduction in a boundary layer by a local spanwise oscillating surface,” Phys. Fluids 6, 3218-3220 (1994).

${ }^{10}$ K.-S. Choi, J.-R. DeBisschop, and B. R. Clayton, "Turbulent boundarylayer control by means of spanwise-wall oscillation,” AIAA J. 36, 1157-1163 (1998).

${ }^{11}$ M. Quadrio and P. Ricco, "Critical assessment of turbulent drag reduction through spanwise wall oscillations," J. Fluid Mech. 521, 251-271 (2004).

${ }^{12}$ E. Touber and M. A. Leschziner, "Near-wall streak modification by spanwise oscillatory wall motion and drag-reduction mechanisms," J. Fluid Mech. 693, 150-200 (2012).

${ }^{13}$ L. Agostini, E. Touber, and M. A. Leschziner, "Spanwise oscillatory wall motion in channel flow: Drag-reduction mechanisms inferred from DNS-predicted phasewise property variations at $\operatorname{Re}_{\tau}=1000$," J. Fluid Mech. 743, 606-635 (2014).

${ }^{14}$ L. Agostini, E. Touber, and M. A. Leschziner, "The turbulence vorticity as a window to the physics of friction-drag reduction by oscillatory wall motion," Int. J. Heat Fluid Flow 51, 3-15 (2015).

${ }^{15} \mathrm{M}$. Quadrio, P. Ricco, and C. Viotti, "Streamwise-travelling waves of spanwise wall velocity for turbulent drag reduction,” J. Fluid Mech. 627, 161-178 (2009).

${ }^{16}$ F. Auteri, A. Baron, M. Belan, G. Campanardi, and M. Quadrio, "Experimental assessment of drag reduction by traveling waves in a turbulent pipe flow," Phys. Fluids 22, 115103 (2010).

${ }^{17}$ K. Gouder, M. Potter, and J. F. Morrison, "Turbulent friction drag reduction using electroactive polymer and electromagnetically driven surfaces," Exp. Fluids 54, 1441 (2013).

${ }^{18}$ J. Bird, M. Santer, and J. F. Morrison, "Experimental control of turbulent boundary layers with in-plane travelling waves," Flow, Turbul. Combust. 100, 1015-1035 (2018).

${ }^{19} \mathrm{M}$. Quadrio, "Drag reduction in turbulent boundary layers by in-plane wall motion,” Philos. Trans. R. Soc., A 369, 1428-1442 (2011).

${ }^{20}$ H. Choi, P. Moin, and J. Kim, “Active turbulence control for drag reduction in wall-bounded flows,” J. Fluid Mech. 262, 75-110 (1994).

${ }^{21}$ T. I. Józsa, E. Balaras, M. Kashtalyan, A. G. L. Borthwick, and I. M. Viola, "Active and passive in-plane wall fluctuations in turbulent channel flows," J. Fluid Mech. 866, 689-720 (2019).

${ }^{22}$ T. I. Józsa, "Drag reduction by passive in-plane wall motions in turbulent wallbounded flows," Ph.D. thesis, University of Edinburgh, 2018.
${ }^{23}$ D. J. Wise, C. Alvarenga, and P. Ricco, "Spinning out of control: Wall turbulence over rotating discs," Phys. Fluids 26, 125107 (2014).

${ }^{24}$ J. Jiménez and A. Pinelli, “The autonomous cycle of near-wall turbulence," J. Fluid Mech. 389, 335-359 (1999).

${ }^{25}$ B. J. Balakumar and R. J. Adrian, "Large- and very-large-scale motions in channel and boundary-layer flows," Philos. Trans. R. Soc., A 365, 665-681 (2007).

${ }^{26}$ R. Mathis, N. Hutchins, and I. Marusic, "Large-scale amplitude modulation of the small-scale structures in turbulent boundary layers," J. Fluid Mech. 628, 311-337 (2009).

${ }^{27}$ B. Ganapathisubramani, N. Hutchins, J. P. Monty, D. Chung, and I. Marusic, "Amplitude and frequency modulation in wall turbulence," J. Fluid Mech. 712, 61-91 (2012).

${ }^{28}$ M. Guala, S. E. Hommema, and R. J. Adrian, "Large-scale and very-large-scale motions in turbulent pipe flow," J. Fluid Mech. 554, 521-542 (2006).

${ }^{29} \mathrm{Y}$. Wu, "A study of energetic large-scale structures in turbulent boundary layer," Phys. Fluids 26, 045113 (2014).

${ }^{30}$ Y. Chang, S. S. Collis, and S. Ramakrishnan, "Viscous effects in control of nearwall turbulence," Phys. Fluids 14, 4069-4080 (2002).

${ }^{31}$ M. R. Abbassi, W. J. Baars, N. Hutchins, and I. Marusic, "Skin-friction drag reduction in a high-Reynolds-number turbulent boundary layer via real-time control of large-scale structures," Int. J. Heat Fluid Flow 67, 30-41 (2017).

${ }^{32}$ K.-S. Choi, "Near-wall structure of turbulent boundary layer with spanwise-wall oscillation," Phys. Fluids 14, 2530-2542 (2002).

${ }^{33}$ H. Schlichting and K. Gersten, Boundary-Layer Theory (Springer, 2016).

${ }^{34}$ S. Liao, Beyond Perturbation: Introduction to the Homotopy Analysis Method (CRC Press, 2003).

${ }^{35}$ H. O. G. Benschop and W.-P. Breugem, "Oscillatory laminar shear flow over a compliant viscoelastic layer on a rigid base," e-print arXiv:1705.04479 [physics.fludyn] (2017); accessed on 26 April 2019.

${ }^{36}$ S. Kyritsi-Yiallourou and G. C. Georgiou, "Newtonian Poiseuille flow in ducts of annular-sector cross-sections with Navier slip," Eur. J. Mech.: B/Fluids 72, 87-102 (2018).

${ }^{37}$ H. O. G. Benschop, A. J. Greidanus, R. Delfos, J. Westerweel, and W.-P. Breugem, "Deformation of a linear viscoelastic compliant coating in a turbulent flow," J. Fluid Mech. 859, 613-658 (2019).

${ }^{38}$ C. Y. Wang, "Exact solutions of the unsteady Navier-Stokes equations," Appl. Mech. Rev. 42, S269-S282 (1989).

${ }^{39}$ C. Y. Wang, "Exact solutions of the steady-state Navier-Stokes equations," Annu. Rev. Fluid Mech. 23, 159-177 (1991).

${ }^{40}$ R. A. Berker, "Sur quelques cas d'intégration des équations du mouvement d'un fluide visqueux incompressible," Ph.D. thesis, University of Lille, Paris-Lille, 1936. ${ }^{41} \mathrm{R}$. Berker, "Intégration des équations du mouvement d'un luide visqueux incompressible," in Handbuch der Physik (Springer Verlag, 1963), pp. 1-384.

${ }^{42} \mathrm{~T}$. K. Sengupta, Instabilities of Flows and Transition to Turbulence (CRC Press, 2012).

${ }^{43}$ P. T. Nagy and G. Paál, "On the sensitivity of planar jets," Int. J. Heat Fluid Flow 62, 114-123 (2016).

${ }^{44}$ P. T. Nagy and G. Paál, "Modeling the perturbation growth in an acoustically excited plane jet," Phys. Fluids 29, 114102 (2017).

${ }^{45}$ J. R. Womersley, "Method for the calculation of velocity, rate of flow and viscous drag in arteries when the pressure gradient is known," J. Physiol. 127, 553-563 (1955).

${ }^{46}$ D. J. Acheson, Elementary Fluid Dynamics (Oxford University Press, 1990).

${ }^{47}$ H. S. Carslaw and J. C. Jaeger, Conduction of Heat in Solids (Oxford Science Publications, 1959).

${ }^{48}$ F. S. Godeferd and F. Moisy, "Structure and dynamics of rotating turbulence: A review of recent experimental and numerical results," Appl. Mech. Rev. 67, 030802 (2015).

${ }^{49}$ A. J. Faller, "An experimental study of the instability of the laminar Ekman boundary layer,” J. Fluid Mech. 15, 560-576 (1963).

${ }^{50}$ R. J. Lingwood, "Absolute instability of the Ekman layer and related rotating flows,” J. Fluid Mech. 331, 405-428 (1997).

${ }^{51}$ F. Marcotte, E. Dormy, and A. Soward, “On the equatorial Ekman layer," J. Fluid Mech. 803, 395-435 (2016). 
${ }^{52} \mathrm{~J}$. Kim and P. Moin, "Application of a fractional-step method to incompressible Navier-Stokes equations,” J. Comput. Phys. 59, 308-323 (1985).

${ }^{53}$ L. Könözsy and D. Drikakis, "A unified fractional-step, artificial compressibility and pressure-projection formulation for solving the incompressible Navier-Stokes equations," Commun. Comput. Phys. 16, 1135-1180 (2014).

${ }^{54}$ M. Szőke, T. I. Józsa, Á. Koleszár, I. Moulitsas, and L. Könözsy, "Performance evaluation of a two-dimensional lattice Boltzmann solver using CUDA and PGAS UPC based parallelisation," ACM Trans. Math. Software 44, 1 (2017).

${ }^{55}$ T.-R. Teschner, L. Könözsy, and K. W. Jenkins, "A generalised and lowdissipative multi-directional characteristics-based scheme with inclusion of the local Riemann problem investigating incompressible flows without free-surfaces," Comput. Phys. Commun. 239, 283 (2019).
${ }^{56}$ C. D. Andereck, S. S. Liu, and H. L. Swinney, "Flow regimes in a circular Couette system with independently rotating cylinders," J. Fluid Mech. 164, 155-183 (1986).

${ }^{57}$ R. A. Verschoof, A. K. te Nijenhuis, S. G. Huisman, C. Sun, and D. Lohse, "Periodically driven Taylor-Couette turbulence," J. Fluid Mech. 846, 834-845 (2018).

${ }^{58}$ M. Seddighi, S. He, P. Orlandi, and A. E. Vardy, "A comparative study of turbulence in ramp-up and ramp-down unsteady flows," Flow, Turbul. Combust. 86, 439-454 (2011).

${ }^{59}$ L. R. J. Sundstrom and M. J. Cervantes, "The self-similarity of wallbounded temporally accelerating turbulent flows," J. Turbul. 19, 49-60 (2018). 